\title{
A study to assess the knowledge and practice regarding aseptic technique adopted during labour among staff nurses
}

\author{
Verma S.R. ${ }^{1}$, Yadav A. ${ }^{2}$, Narwal R. ${ }^{3}$ \\ ${ }^{1}$ Mr. Shiv Ram Verma, Nursing Tutor, GNMTC, Govt. S. K. Hospital, Sikar, ${ }^{2}$ Dr. Ashok Yadav, Nursing Lecturer, \\ Government College of Nursing, Jaipur, ${ }^{3}$ Mrs. Ritu Narwal, Nursing Lecturer, Vinayak College of Nursing, Kalwar \\ Road, Jaipur, Rajasthan, India
}

Corresponding Author: Mr. Shiv Ram Verma, Nursing Tutor, GNMTC, Govt. S.K. Hospital, Sikar, Rajasthan, India. E-mail: shivram.jaipur82@gmail.com

\begin{abstract}
Background: Aseptic technique is a set of specific practices and procedures performed control condition with the goal of minimizing sepsis. Pathogens may introduce infection through contact with the environment, personal or equipment. Objectives: To assess the knowledge and practice on aseptic technique adopted during labour among staff nurses and also compare existing knowledge and practice. Methods: It is a non- experimental descriptive study carried out on the staff nurses working in labour rooms of the hospitals of Sikar, Rajasthan. A study was conducted during the year of 2018 among 50 staff nurses working in labour rooms of the hospitals, who were selected by using non-probability two stage cluster sampling technique. A self-administered structured questionnaire and an observation checklist were used for data collection. Result: The findings indicated that majority of 37 (74\%) staff nurses had fair knowledge and 36 (72\%) had fair attitude regarding aseptic technique adopted during labour. The mean of knowledge and practice test (14.42 \& 19.04) and SD of knowledge and practice test was (3.62 \& 4.65). There was significant association between the knowledge and practice. Conclusion: The study concluded that the good knowledge and practice regarding aseptic technique adopted during labour is the key issue for better maternal health.
\end{abstract}

Key words- Aseptic technique, Labour, Staff nurse and Labour room.

\section{Introduction}

The concept of asepsis can be applied in any clinical setting. Typical situation that call for aseptic measures include surgery, insertion of intravenous line, urinary catheter and labour room setting [1]. Strict asepsis during the delivery are practices "no touch technique" which is any instrument which is to be inserted in the cervical canal much not touch any non-sterile object or surface prior to insertion. Sterilization or high level disinfection of instrument with meticulous attention should be followed in the labour rooms [2].

Aseptic techniques are employed to maximize and maintain asepsis, the absence of pathogenic organisms, in the clinical setting. A key element requiring careful attention is equipment or supplies Medical equipment can be sterilize by chemical, treatment, radiation and gas or heat.

Manuscript received: $14^{\text {th }}$ November 2018

Reviewed: $24^{\text {th }}$ November 2018

Author Corrected: $30^{\text {th }}$ November 2018

Accepted for Publication: $6^{\text {th }}$ December 2018
Personal can take steps to ensure sterility by assessing that sterile packages are dry and intact and checking sterility indicators such as dates or colored tape that changes color when sterile [3].

The universal precaution to control infection which applied by all medical and para-medical staffs include the basic elements such as hand washing thoroughly with soap and water before caring out the procedure, immediately. In case autoclaving is not possible, the instrument must be fully immersed in water in a covered container and boiled for at least 20 minutes. Infection Control is a most important field to concern in labor and delivery room as the newborn babies takes time to adapt to their surroundings, after immediately coming out of their mother womb. Neonatal infections may be acquired by trans-placental transfer or during delivery in birth canal or other means during postpartum. Infection control is the policies and procedures used to reduce the infection transmission risk especially in hospitals and health care settings. 


\section{Original Research Article}

Neonates and children are more prone to acquired infections, precautionary measures has to be taken to reduce the chances of getting infection. Conducting deliveries in unhygienic places or not properly disinfected labor rooms increases the risk of spreading infections form mother to baby or from one person to another person.

If health care personnel and health care units authorities are not adhere to infection prevention protocols in labor room then maternal and neonatal infections rises which in turn leads to increase maternal and infant mortality rate, also economic loss to community [2].

A protective and aseptic environment in the hospital unit is very essential as a prerequisite particularly services provided in the labour room. There are many studies conducted in India and other countries on this issue and findings show that many staff nurse's fails to perform good practice and have poor knowledge on aseptic technique in labour room so researcher select the present study for assessing knowledge, practice and compare both on aseptic technique in labour room and further analysis of study.

\section{Material and Methods}

Place of study: It study carried out in labour rooms of the hospitals of Sikar, Rajasthan during the year of 2018.

Type of study: It is a non-experimental descriptive study.

Sample and sampling methods: The study was conducted among 50 staff nurses working in labour rooms of the hospitals, who were selected by using nonprobability two stage cluster sampling technique.
Data collection instrument: A self-administered structured knowledge questionnaire and an observation checklist were used for data collection. The data collection tool comprises 2 tools; Tool- I included 28 items to assess knowledge regarding aseptic technique adopted during labour by staff nurses. Tool-II (Observation checklist) included total 36 items regarding aseptic technique adopted during labour by staff nurses, in which 17 included in preparation aspect, 9 in assisting the birth aspect and 10 in AMTSL aspect of labour.

Pilot study: A pilot study was performed on 5 staff nurses working in labour rooms of hospitals to test the reliability and feasibility of study. These subjects were not included in the main study. Content validity of the tool was established by giving to professional expert and reliability was established by Kuder-Richardson ( $\mathrm{r}$ KR-20) and Cohen's Kappa measure.

Scoring method: Total score for knowledge questionnaire was 28 and it was 36 for observation checklist. Score 'One' for each correct response and score 'Zero' for each incorrect response. Staff nurses were distributed into poor (score $\leq 33 \%$ ), fair (score between 33-66\%) and good (score above 66\%) knowledge (total score 28) and attitude (total score 36) on the basis of secured knowledge and attitude score in knowledge test and in non-participatory observation schedule.

Statistical method: Collected Data was organized and descriptive statistics was used to calculate mean, mean $\%$, median and standard deviation. Karl-Pearson correlation coefficient formula was applied for finding significant relationship between knowledge and practice of staff nurses and to test hypothesis at 0.05 level of significance.

\section{Results}

The findings are summarized as follows:

1. Majority of respondents $37(74 \%)$ had fair knowledge, 4 (8\%) had poor knowledge and only 9 (18\%) had good knowledge on aseptic technique adopted during labour. Mean knowledge score (mean=14.42) mean\% $(51.43 \%)$ and standard deviation $(\mathrm{SD}=3.62)$.

2. Majority of respondents $36(72 \%)$ performed fair practice, $6(12 \%)$ performed poor practice and only 8 (16\%) performed good practice on aseptic technique adopted during labour. Mean attitude score obtained by the respondents was $57.29 \%$ with SD of 4.41 in preparation before labour aspect, mean percentage obtained by the respondents was $47.33 \%$ with SD of 2.51 in assisting the birth aspect, mean percentage obtained by the respondents was $50.4 \%$ with SD of 3.19 in AMTSL aspect and overall mean percentage obtained by the respondents was $52.89 \%$ with SD of 4.65 regarding the aseptic technique adopted during labour.

3. Findings shows that $8 \%$ staff nurses had poor knowledge and $12 \%$ staff nurses had poor practice regarding aseptic technique adopted during labour. 
4. The study shows that there was significant association between the knowledge and practice. So null hypothesis is rejected and researcher accepts the research hypothesis at 0.05 level of significance.

Table-1: Distribution of staff nurses by the level of knowledge $\mathbf{N}=\mathbf{5 0}$

\begin{tabular}{|l|c|c|c|}
\hline Level of Knowledge & Score & Frequency & Percentage \\
\hline Poor Knowledge $(\leq 33 \%)$ & $\leq 9$ & 4 & $8 \%$ \\
\hline Fair Knowledge $(\leq 33-66 \%)$ & $10-18$ & 37 & $74 \%$ \\
\hline Good Knowledge (Above 66 \%) & $>18$ & 9 & $18 \%$ \\
\hline Total & $\mathbf{2 8}$ & $\mathbf{5 0}$ & $\mathbf{1 0 0 \%}$ \\
\hline
\end{tabular}

Table-2: Aspect wise Practice score of staff nurses on aseptic technique adopted during labour $\mathbf{N}=\mathbf{5 0}$

\begin{tabular}{|l|c|c|c|c|c|}
\hline Aspect & $\begin{array}{c}\text { Max. } \\
\text { Score }\end{array}$ & Mean & Median & $\begin{array}{c}\text { Mean } \\
\text { \% }\end{array}$ & SD \\
\hline Practice on aseptic technique adopted during preparation & 17 & 9.74 & 10 & 57.29 & 4.41 \\
\hline Practice on aseptic technique adopted during Assisting the birth & 9 & 4.26 & 4 & 47.33 & 2.15 \\
\hline Practice on aseptic technique adopted during AMTSL & 10 & 5.04 & 5 & 50.4 & 3.19 \\
\hline Overall Practice on aseptic technique adopted during labour & 36 & 19.04 & 19 & 52.89 & 4.65 \\
\hline
\end{tabular}

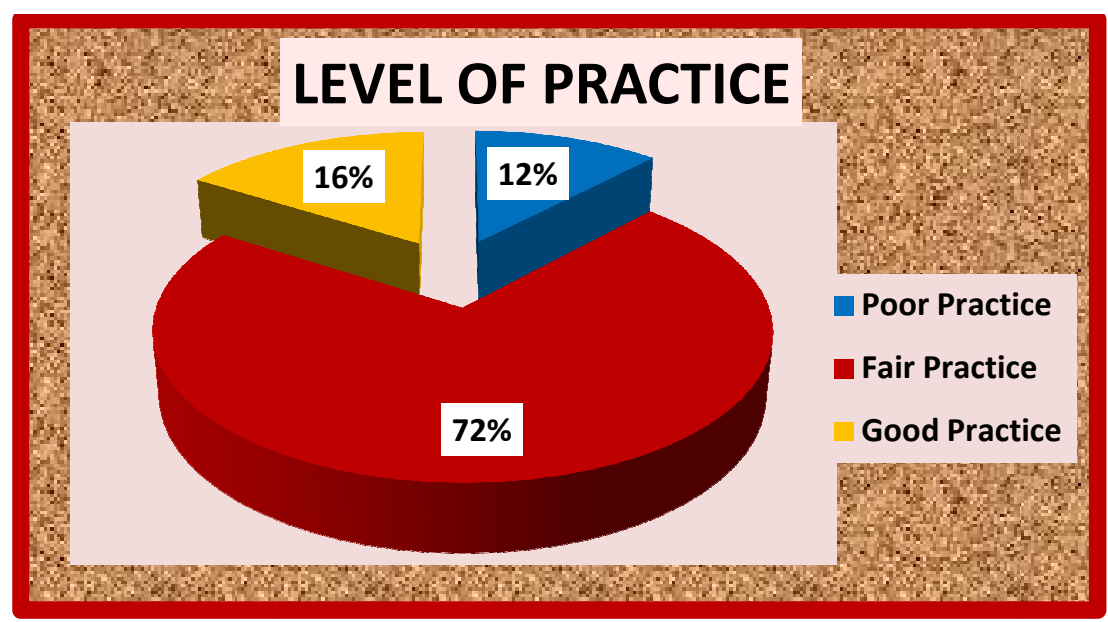

Figure-1: Distribution of staff nurses by the level of Practice

\section{Discussion}

The present study is an effort to assess the knowledge, practice and comparison between these technique adopted in labour rooms of hospital among staff nurses. In order to achieve the objective of the study a nonexperimental descriptive research design was adopted. 50 staff nurses were selected by using non-probability two stage cluster sampling technique, fulfilling the criteria of sample.

Finding of the present study revealed that majority of 37 (74\%) staff nurses had fair knowledge, $4(8 \%)$ had poor knowledge and $36(72 \%)$ had fair attitude and $6(12 \%)$ performed poor practice. Mean knowledge score (mean=14.42) mean \% $(51.43 \%)$ and standard deviation $(\mathrm{SD}=3.62)$ and mean attitude obtained by the respondents was $57.29 \%$.
The finding of present study supported by study conducted by Barkha Devi and ReshmaTamang on Knowledge and Practice of Aseptic Technique During Delivery Among Health Professionals in Selected Government hospitals of Sikkim and published in 2014, The findings of the study reveals that knowledge regarding Bio-medical waste management (87\%), definition of asepsis, aseptic technique, infection (86\%), aseptic technique in labour room $(77 \%)$ and asepsis in stages of labour $(59 \%)$ was found to be better whereas asepsis during delivery $(57 \%)$ was found to be less. The knowledge regarding asepsis during delivery needs more emphasis [4]. The finding of present study supported by study conducted by Navjyot Singh, Manisha Rani et al., on Assessment of Knowledge of Staff Nurses Regarding Aseptic Technique, 68 staff 


\section{Original Research Article}

nurses selected by convenient sampling technique from selected hospital of of Pilkhuwa, Hapur. Findings of study showed that mean knowledge score of staff nurses was 13.13 with median score of 14 . Maximum staff nurses i.e. $43(63.24 \%)$ were having average knowledge whereas $25(36.76 \%)$ having knowledge poor average, none of the staff nurse was having adequate knowledge regarding aseptic technique [5].

The finding of present study supported by study conducted by Jothi Bala (2009) on knowledge and practice of staff nurses regarding infection control in $\mathrm{MCH}$ area of selected hospitals, in Ludhiana, Punjab. A sample of 60 staff nurses were selected purposively. The final result depicted that staff nurses had efficient knowledge on infection control $(80 \%)$ whereas level of practice were not appropriate to the standard [6].

The finding of present study supported by study conducted by Benita D, Vijayalakshmi et al., to evaluate the effectiveness of Infection Control Standards on Practice among Health Care Personnel Working in Labour Unit at selected hospitals, Nagercoil. 60 health care personnel were selected as samples using purposive sampling technique. Quantitative research approach with Pre-experimental one group pre test post test design was used to collect the data.

Data was collected by using observational checklist consisted of 4 components to assess the level of practice on infection control among the health care personnel working in labour unit. The findings of the study revealed that the mean pretest level of practice on infection control was 21.47 with standard deviation of 1.92 and mean post test level of practice on infection control was 43.73 with standard deviation of 2.02. The result revealed that there was an deficiency of practice and need to improve among health care personnel [7].

Dr. Shreenivas Shouri1 and Dr. Suchitra R, Department of Obstetrics \& Gynecology, Government Medical College, Ananthapuram, Andhra Pradesh conducted a study to assess the efficacy of infection control practices various factors were considered and analyzed before and after implementation of infection control policies and practices.

Various aspects observed were environmental surveillance reports, clinically suspected or confirmed infections of patients in labor, needle stick injuries incidence, puerperal infections rate, neonatal infections and hand hygiene compliance. These infection control indicator rates were collected and analyzed. Puerperal and Neonatal infections were noted as 39\% and 34\% before, $17 \%$ and $13 \%$ after introducing proper infection control guidelines. Hand hygiene compliance decreased from $26 \%$ to $8 \%$ after introducing mandatory hand hygiene guidelines. $37 \%$ and $74 \%$ were using PPE according to protocol before and after implementing protocols respectively. All health care personnel are required to adhere to standard precautions and infection control guidelines to avoid transmission of infections [8].

The finding of present study was supported by crosssectional study conducted by Seyed Mehdi Tabatabaei, Fateme Behmanesh Pour et al., to investigate infection control program management and midwives' practices in labor and delivery units. Data was collected from 88 midwives in four labor and delivery units in public hospitals in Zahedan, southeast Iran.

The evaluation scores for all aspects of infection control were suboptimal; infection control program management $38.1 \%$ to $71.4 \%$, healthcare workers postexposure measures $58.5 \%$ to $92.7 \%$, medical waste management $73.9 \%$ to $87.0 \%$ and infection control related standard infrastructures and equipments $55.9 \%$ to $82.8 \%$.

The midwives mean scores for attending infection control training courses and hand hygiene were less than $40 \%$ of the maximum score, but the mean scores for normal vaginal deliveries scrub, equipment and instruments hygiene practices were generally above $70 \%$. The results of this study revealed a need for development of appropriate policies and protocols for infection control practices in labor and delivery units and also midwives training on clean delivery practices [9].

The finding of present study was supported by descriptive quasi-experimental research begin in $20^{\text {th }}$ February to $26^{\text {th }}$ May 2016 by Wafa A K Abbas, Muntahaa Rashaan, Faculty of Nursing, University of Babylon to assess hand hygiene practices of health care personnel in the delivery room at the middle Euphrates teaching hospitals and involve all midwives and physicians in the delivery room.

The study indicate that the overall evaluation for the health staff practices regarding hand hygiene is fair at Karbala, Al-Najaf, Babylon and Diwaniah with high difference in health staff practices regarding infection control precautions (hand hygiene). Also proved that Hand hygiene is the most important and effective 


\section{Original Research Article}

infection prevention and control measure to prevent the spread of microorganisms causing HAIs and improving hand hygiene is consider a vital intervention to promote optimum patient safety in delivery room [10].

The finding of present study was supported by study conducted by Nadia Abdalla \& Afaf Salah Abed ElMohsen on Effectiveness of infection control standers on practice among Health care Personnel working in $\mathrm{MCH}$ centers at Quena Governorate, all nurses working in four $\mathrm{MCH}$ center were included in the study include nurses.

It included two tools and interviewing questioner to assess socio demographic characteristics of the nurses and nurses' knowledge about universal precautions and infection control precautions before, during and after normal vaginal labor and observational checklist to assess nurses' performance related to application of universal precautions before, during and after normal vaginal delivery.

The scores of nurses' knowledge and performance having Bachelor of Science in nursing were significantly higher than those who having of nursing Diploma. Study recommended that Periodic training program should be provided to nurses at $\mathrm{MCH}$ centers to update their knowledge and practices regarding universal infection control precautions in labour room [11].

The finding of present study was supported by a Crosssectional study conducted by Friday O, Edoja O et al., for Assessment of infection control practices in maternity units in Southern Nigeria, study consisting of in-depth interviews with service providers, observation of clinical practices and examination of medical records. Data was collected from 63 health facilities of primary, secondary and tertiary maternity care centers.

Three pre-tested tools were adapted to the local setting and used to interview key informants in the health facilities and to observe for practices and records relating to infection control. Of the 63 health facilities, $68 \%$ (43) reported that they had infection control procedures in place, while $33 \%$ (21) reported that they had an ongoing programme for staff training on infection control. A high proportion of the health facilities reported that staffs routinely wash their hands before and after sterile procedures, but only half of the facilities were observed to have 24-h running water and only two-thirds had soap and antiseptic solutions in delivery and operating theatre areas. The results of this study suggest the need for improved staff training on infection control in maternity care facilities in Edo State [12].

The finding of present study was supported by a study conducted by Rajesh Mehta, Dileep V Mavalankar et.al. to provide information on procedures and practices related to infection control in labour and delivery units in Gujarat state, India. Twenty health care facilities, including private and public primary health centres and referral hospitals, were sampled from two districts in Gujarat state, India. Three pre-tested tools for interviewing and for observation were used.

Data collection was based on existing infection control guidelines for clean practices, clean equipment, clean environment and availability of diagnostics and treatment. Seventy percent of respondents said that standard infection control procedures were followed, but a written procedure was only available in $5 \%$ of facilities.

Alcohol rubs were not used for hand cleaning and surgical gloves were reused in over $70 \%$ of facilities, especially for vaginal examinations in the labour room. Most types of equipment and supplies were available but a third of facilities did not have wash basins with "hands-free" taps. Only 15\% of facilities reported that wiping of surfaces was done immediately after each delivery in labour rooms. This study of current infection control procedures and practices during labour and delivery in health facilities in Gujarat revealed a need for improved information systems, protocols and procedures, and for training and research [13].

\section{Conclusion}

The study concluded that the good knowledge and practice regarding aseptic technique adopted during labour is the key issue for better maternal health. Findings of present study revealed that knowledge and practice regarding aseptic technique adopted during labour was poor among at least $10 \%$ of staff nurses working in labour rooms of hospitals.

Contribution by author-Dr. Ashok Yadav sir provided us a continuous supervision and guidance at each and every step throughout the study from selection of problem statement to the final writing of this manuscript. We both actively work in this study in every step such as selection of problem statement, searching of concerning literatures, data collection, framing of manuscript, data analysis and discussion of the findings. 


\section{Original Research Article}

What does this study add in existing knowledge: This study will be helpful for staff nurses to identify the various steps included in different stages of labour and enhance their existing knowledge on aseptic technique adopted during labour by self administered structured knowledge questionnaire.

\section{Funding: Nil, Conflict of interest: None}

Permission of IRB: Yes

\section{References}

1. Bjerke \& Nancy, Hand Hygiene in Health care: Playing by the new rules, Infection Control Today February 2003. Available from: https://www. infectioncontroltoday.com/hand-hygiene/hand-hygienehealthcare-playing-new-rules.

2. Myles Textbook for Midwife $13^{\text {th }}$ Edition. Churchill Livingstone Publishes: 414-425.

3. Osman Cathy. Asepsis and Aseptic Practices in the Operating room, Infection Control Today July 2000. Available from: https://www.infectioncontroltoday. com/hand-hygiene/asepsis-and-aseptic-practicesoperating-room.

4. Barkha Devi and Reshma Tamang, Knowledge on Practice of Aseptic Technique during Delivery among Health Professionals in Selected Government hospitals of Sikkim, Available from: http://medjournal. smu. edu.in/articles/2014july/29.pdf.

5. Navjyot Singh, Manisha Rani and Priyanka. Assessment of Knowledge of Staff Nurses Regarding Aseptic Technique, www. ijhsr.org, September 2016.

6. Jyoti Bala, Knowledge and practices of staff nurses regarding infection control in $\mathrm{MCH}$ area of a selected Hospital Ludhiana, Punjab: 6th International Neonatal Nursing Conference - ICNN, 2007, Sep. P/ICNN/ CIPr/ 21, Pp 69.
7. Lin CF, Yang CY, Lu MS, et al. Effectiveness of a nosocomial infection control training in improving knowledge in patient-hired attendants and outsourced workers in Taiwan. J Nurs Res. 2008 Sep;16(3):187-94.

8. Shreenivas Shouri1 and Suchitra R, A study on Prevention of transmission of infections in labor room by implementing proper sterility techniques, IOSR Journal of Dental and Medical Sciences.

9. Seyed Mehdi Tabatabaei, Fateme Behmanesh Pour and Hannaneh Azadeh, A Cross-Sectional Study on Infection Control Practices and Program Management in Labor and Delivery Units. Available from: http://intjinfection.com/en/articles/14739.html

10. Wafa A K Abbas, Muntahaa Rashaan, Health Personnel Practices Regarding Hand Hygiene in the Delivery Room at the Middle Euphrates Teaching Hospitals, www.ijpcr.com

11. Nadia Abdalla \& Afaf Salah Abed El-Mohsen conducted a study on Effectiveness of infection control standers on practice among Health care Personnel, IOSR Journal of Nursing and Health Science, Jul. Aug. 2015, Available from: https:// pdfs. semanticscholar.org /a4b7/a57bd01db51c62b208d b81e 0c1cf7b08e379 pdf.

12. Friday O, Edoja O, Osasu A, et al. Assessment of infection control practices in maternity units in Southern Nigeria. Int J Qual Health Care. 2012 Dec;24(6):634-40. doi: 10.1093/intqhe/mzs057. Epub 2012 Oct 18 .

13. Mehta R, Mavalankar DV, Ramani KV, et al. Infection control in delivery care units, Gujarat state, India: a needs assessment. BMC Pregnancy Childbirth. 2011 May 20;11:37. doi: 10.1186 /1471-2393-11-37.

\section{How to cite this article?}

Verma S.R., Yadav A., Narwal R. A study to assess the knowledge and practice regarding aseptic technique adopted during labour among staff nurses. Int J Med Res Rev 2018; 6(08): 487-492. doi:10.17511/ijmrr.2018.i08.14. 